\title{
NON-ALCOHOLIC STEATOHEPATITIS IN PATIENTS WITH OBESITY: INTERCONNECTION BETWEEN BODY MASS, CHANGES OF LIPID METABOLISM AND CONCOMITANT PATHOLOGY OF BILIARY TRACT
}

\author{
Aleksandra Filippova \\ Department of Internal Medicine № 2 \\ SE «Dnipro Medical Academy of Health Ministry of Ukraine» \\ 9 Dzerzhinsky str., Dnepropetrovsk, Ukraine, 49044 \\ Filippova-dma@i.ua
}

\begin{abstract}
Aim. To study the main indices of lipid blood spectrum, which changes lead to DL formation, depending of body mass index (BMI) and concomitant pathology of biliary tract in patients with NASH and obesity.

Materials and methods. There were examined 100 patients with NASH in combination with obesity and BT pathology, in which during sonographic or morphological study of bioptat of the liver were revealed the signs of hepatic steatosis. Among patients were 40 men and 60 women. The mean age of patients - $(54,68 \pm 1,15)$ years old. The control group included 20 practically healthy persons (PHP).

BMI was determined by Quetelet formula. Depending of the degree of BMI increase all patients with NASH and obesity were divided into three groups: 1 group - with BMI 25-29,9 kg/m² - EBM; 2 group - with BMI 30-34,9 kg/m² - obesity of I degree; 3 group - with BMI 35-39,9 kg/m² - obesity of II degree. All examined patients had concomitant pathology from BT side. Depending on BT pathology all patients were also divided into 3 groups: NASH with chronic non-calculous cholecystitis (CNC); NASH with chronic calculous cholecystitis (CCC); NASH in patients after laparoscopic cholecystectomy (LCE) with concomitant postcholecystectomical syndrome (PCES). For characteristic of lipid exchange were determined the general cholesterol (GCS), general lipids (GL), triglycerides (TG), lipoproteins of the low density (LDL), lipoproteins of the high density (HDL). DL phenotype was determined by D. S. Fredrickson classification with modern additions.

Results. Analysis of lipid changes in patients with NASH demonstrated the reliable increase of GCS with parallel rise of TG, GL, LDL, fixed at increase of BMI relative to PHP $(p<0,05, p<0,001)$ and to patients of other groups ( $<<0,05)$. Maximal values of GCS, TG, GL and also LDL were determined in 2 and 3 groups of observation at obesity of I and II degrees. There was registered the reliable decrease of HDL content in most patients of 3 group relative to the ones of 2 group, $(p<0,01)$ and of 1 one.

The level of GCS, TG, GL and LDL was almost equally increased in all groups of observation of NASH: with concomitant $\mathrm{CNC}, \mathrm{CCC}$ and in patients with PCES relative to PSP, $(\mathrm{p}<0,001)$. HDL indices were equally decreased at concomitant $\mathrm{CNC}$ and $\mathrm{CCC}$ relative to the patients with PCES background with HDL level within physiological norm $\left(\mathrm{p}<0,001, \mathrm{p}_{\mathrm{F}}=0,009\right)$.

There are separated two main phenotypes of DL: IIa and IIb that were typical for patients with NASH and obesity with BT pathology. It was established, that IIb phenotype of dyslipidemia was most often observed in patients with BMI $30-34,9 \mathrm{~kg} / \mathrm{m}^{2}$ and $35-39,9 \mathrm{~kg} / \mathrm{m}^{2}$.

Discussion. There were determined the features of changes of lipid blood spectrum of in this category of patients that are manifested by variability of disorders from hypercholesterolemia and hypertriglyceridemia on the background of increase of GCS, TG, GL, LDL and decrease of HDL. The progression of lipid disorders in patients with NASH in combination with obesity and BT pathology depends on increase of BMI parameters.

The concomitant pathology from BT side partially influenced the indices of lipid blood spectrum. Thus, the low level of HDL in patients with NASH in combination with obesity and concomitant CNC and CCC can be explained by the intense inflammatory processes in BT, attended with disorder of indices of lipid profile with decrease of HDL and increase of GCS, TG, GL, LDL.

Conclusion. Progression of intensity of lipid disorders in patients with NASH in combination with obesity and BT pathology depends on increase of BMI parameters.

The concomitant BT pathology partially influences the parameters of lipid blood spectrum. HDL was equally decreased in all patients with NASH at CNC and CCC relative to patients with concomitant PCES and HDL level within physiological norm. At NASH in combination with obesity at increase of BMI the DL phenomena more often appear in patients. There are separated two main phenotypes of dyslipidemias: IIa and IIb.
\end{abstract}

Keywords: non-alcoholic steatohepatits, obesity, biliary tract, lipid metabolism, body mass index. 


\section{Introduction}

Liver plays an important role for development of lipid metabolism with formation of dyslipidemia (DL). According to the literature data, DL (hypertriglyceridemia, hypercholesterolemia or their combination) are often observed at fatty disease of liver - in 20-81\% of cases [1]. Today the term "dyslipidemia" is connected with diverse changes of lipid spectrum in blood plasma - rise, lowering or even complete absence of separate fractions, appearance of unusual or pathological phenotypes [2]. DL is a proved risk of development of NAFDL [3], obesity [4] and cardiovascular pathology [5-7].

The changes of lipid metabolism begin at the level of hepatocyte [8]. Chronic diffuse disease of the liver (CDDL) and namely non-alcoholic fatty disease of the liver (NAFDL) is a specific model of disorder of many mechanisms of lipid metabolism regulation. According to the literature data, the disorders of lipid metabolism are based on disorders of the functional state of liver [9]. It is typical, that in patients with NAFDL at hypertriglyceridemia non-alcoholic steatohepatitis (NASH) develops more often that at hypercholesterolemia [1].

Analysis of literature demonstrates that at excessive body mass (EBM) or obesity is usually observed the certain degree of disorder of lipid, carbohydrate and other metabolisms [10]. Obesity is associated with DL, including the intense hypertriglyceridemia, increase of the content of lipoproteins of very low density, decrease of formation of lipoproteins of the high density (HDL) [11].

At the modern stage the data about disorders of lipid exchange, collected in literature, can form hypothesis, according to which, as a result of human evolution as biological specie was created the specific type of lipid metabolism, at which even the middle (normal) level of cholesterol (CS) is potentially dangerous in the aspect of DL development [3] and, as a consequence, NAFDH [2], cholesterosis of gall bladder, cholelithiasis or adipogenous pancreatitis [12]. It is possible, that the special "tension" of the human lipid metabolism can be the retribution for the way of life: features of nutrition, insufficient physical and intense intellectual activity, non-season hormonal activity [3]. The other important proposition is that the hypercholesterolemia it is not a single factor of disorder of lipid exchange and the risk of NAFDL and obesity development [2].

According to our researchers, the lipids of the blood serum, measured in the different periods of development of hepatobiliary pathology can be the weak reflection of the true DL in the critical time of gallstones creation [1]. The proof of this statement is the following facts: after cholecystectomy the decrease of lipids of blood serum is registered and at exacerbation of chronic calculous cholecystitis, especially, at appearance and increase of concrements the lipids level in blood reliably raises [12].

Thus, for adequate analysis of disorders of lipid metabolism at NAFDL it is important to evaluate not only the level of general cholesterol (GCS) in blood but also the level of lipoproteins of the low density (LDL), HDL, general lipids (GL) and also obligatory the level of triglycerides (TG).

\section{Aim of the work}

To study the main indices of lipid spectrum of blood, which changes lead to the DL formation, depending on body mass index (BMI) and concomitant pathology of biliary tract (BT) in patients with NASH in combination with obesity.

\section{Materials and methods}

There were examined 100 patients with NASH in combination with obesity and BT pathology, who were on stationary treatment in clinic of SI "Institute of gastroenterology, NAMS of Ukraine", in which were detected the sigs of hepatic steatosis during sonographic or morphological study of bioptat of the liver. Among patients were 40 men and 60 women. The mean age of patients $-(54,68 \pm 1,15)$ years old. The control group included 20 practically healthy persons (PHP). All patients signed the informed consent to participation in the research.

Diagnosis of NASH and BT pathology was set according to standardized protocols of diagnostics and treatment of diseases of digestive organs in correspondence with order of MHP of Ukraine № 271 of 13.06.2005 year and № 826 of 06.08 .2014 [13, 14], ICD-10, on the base of the data of anamnesis, clinical and instrumental (ultrasound of organs of abdominal cavity, intraop- 
erational puncture biopsy of the liver) examination, with obligatory taking into account of data of conventional biochemical indices and on the base of increase of the hepatic enzymes level. The intensity of NASH was evaluated by the level of alanine aminotransferase (ALT): minimal at increase of ALT up to two norms, moderate from 3 to 5 norms, high - more than five norms.

Patients involved into research do not overused alcohol (consumption $<50 \mathrm{~g}$ of ethanol/week for men, $<30 \mathrm{~g}$ of ethanol/week for women during last year). In examined persons were not revealed serum markers of viral hepatitis B and C, autoimmune and hereditary disease of liver.

All patients underwent wide anthropometric examination: were weighted fasting, their height was measured, the volume of waist (VW) and hips ( VH) were determined. The index of VW/VH ratio was used for determination of character of fat distribution in organism. Obesity was considered as abdominal one, if in women the value of $\mathrm{VW} / \mathrm{VH}>0,88$, in men $>0,90$ [15]. BMI was determined by the Quetelet formula. Depending of the degree of BMI increase all patients with NASH and obesity were divided into three groups: 1 group - with BMI 25-29,9 kg/m² - EBM; 2 group - with BMI 30-34,9 kg/m² - obesity of I degree; 3 group - with BMI 35-39,9 kg/m² - obesity of II degree. All examined patients had concomitant pathology from BT side. Depending on BT pathology all patients were also divided into 3 groups: NASH with chronic non-calculous cholecystitis (CNC); NASH with chronic calculous cholecystitis (CCC); NASH in patients after laparoscopic cholecystectomy (LCE) with concomitant postcholecystectomical syndrome (PCES). For characteristic of lipid exchange were determined the general cholesterol (GCS), general lipids (GL), triglycerides (TG), lipoproteins of the low density (LDL), lipoproteins of the high density (HDL). The GL and TG content in blood was studied using the sets of "Lachema" biotest, GCS content - by Ilk method, LDL and HDL - according to Burstein and Samay [5].

DL phenotype was determined by D.S. Fredrickson classification with modern additions. DL criteria in examined persons were the levels of GCS $-5,0 \mathrm{mmol} / \mathrm{l}, \mathrm{TG}-1,7 \mathrm{mmol} / \mathrm{l}, \mathrm{HDL}-1,0 \mathrm{mmol} / 1$ in men and 1,2 $\mathrm{mmol} / 1$ in women and LDL 52,5 - units [8].

For diagnostics of hepatic steatosis was used the ultrasound method [3], that give a possibility not only to assess the state of liver but also reveal the deviation of BT, hepatic vessels and pancreas with the help of sonographic system of the linear scanning in the real time "Sonoscope-30" made by Krancbühler. The study was carried out fasting. Analysis of structural changes of digestive organs included assessment of sizes, outlines, acoustic structure and echogenisity of liver and pancreas, state of biliary system by the conventional method [4].

For verification of diagnosis 22 patients underwent intraoperational biopsy of liver with the further study of its histostructure. For histological examination the cuts were colored with hematoxylin and eosin by the method of Mallory-Slinchenko [7]. The state of histostructure of liver was assessed half-quantitatively in the system of stages ( 0 - absent, 1 - insignificant, 3-5\%, 2 - moderate, 3 - intense 60-100\%), the type of cholestasis, degree of chronic inflammation degree of protein, fatty and carbohydrate dystrophy. Histological preparations were studied using the photic microscope „LIUMAM-I2”.

For statistical analysis of data was used STATISTICA 6.1 license program. Qualitative indices, given as a mean value and standard error of mean $(\mathrm{M} \pm \mathrm{m})$. For comparison of mean indices in all subgroups was used the Student's criterion and ANOVA univariate disperse analysis; for relative indices - Pirson's Xi-square criterion $\left(\chi^{2}\right)$. For assessment of interconnection between signs correlative analysis with calculation of coefficients of Spearman's range correlation was realized $\left(\mathrm{r}_{\mathrm{s}}\right)$.

\section{Results}

Analysis of lipid changes in patients with NASH (Table 1) demonstrated the reliable increase of GCS with parallel increase of TG, GL, LDL, fixed at the growth of BMI, relative to PHP (from $\mathrm{p}<0,05$ to $\mathrm{p}<0,001$ ) and patients of other groups $\mathrm{p}<0,05$ ). GCS in patients with NASH was $8,69 \pm 0,55$ in 3 group and 7,77 $\pm 0,36 \mathrm{mmol} / 1$ in 2 group, with increase by $35,6 \%$ and $21,2 \%$ respectively relative to the patients of 1 group $(6,41 \pm 0,49 \mathrm{mmol} / \mathrm{l} ; \mathrm{p}<0,05)$ and with increase in 1,7 and 1,5 times as to PHP ( $\mathrm{p}<0,001$ at all comparisons). The maximal values of TG were also detect-


increase by $38,3 \%$ and $31,4 \%$ comparing with 1 group with TG level $1,88 \pm 0,15 \mathrm{mmol} / \mathrm{l}(\mathrm{p}<0,05)$ 
and with decrease in 2,3 and 2,2 times as to PHP ( $p<0,001$ at all comparisons). The level of GL maximally changed in patients of 3 group $(9,54 \pm 0,31 \mathrm{~g} / 1)$, exceeding the respective values in PHP in 1,8 times $(\mathrm{p}<0,001)$, in patients of 1 group $(7,31 \pm 0,33 \mathrm{~g} / \mathrm{l})$ by $30,5 \%(\mathrm{p}<0,001)$, indices in 2 group $(8,41 \pm 0,26 \mathrm{~g} / \mathrm{l})-$ by $13,4 \%(\mathrm{p}<0,01)$.

Table 1

Comparative characteristic of indices of lipid metabolism in patients with non-alcoholic steatohepatitis in combination with obesity and biliary tract pathology depending on BMI $(\mathrm{M} \pm \mathrm{m})$

\begin{tabular}{|c|c|c|c|c|c|}
\hline \multirow[b]{2}{*}{ Index } & \multirow[b]{2}{*}{ PHP, $n=20$} & \multicolumn{4}{|c|}{ NASH, $n=100$} \\
\hline & & $\begin{array}{c}1 \text { group, } \\
\text { BMI }\left(25-29,9 \mathrm{~kg} / \mathrm{m}^{2}\right), \\
\mathrm{n}=40\end{array}$ & $\begin{array}{c}2 \text { group, } \\
\text { BMI }\left(30-34,9 \mathrm{~kg} / \mathrm{m}^{2}\right), \\
\text { n=37 }\end{array}$ & $\begin{array}{c}3 \text { group, } \\
\text { BMI }\left(35-39,9 \mathrm{~kg} / \mathrm{m}^{2}\right), \\
\mathrm{n}=23\end{array}$ & $\mathbf{p}_{\mathrm{F}}$ between groups \\
\hline GCS, mmol/1 & $5,23 \pm 0,17$ & $6,41 \pm 0,49 *$ & $7,77 \pm 0,36 * * \#$ & $8,69 \pm 0,55^{* * \#}$ & 0,005 \\
\hline $\mathrm{TG}, \mathrm{mmol} / \mathrm{l}$ & $1,14 \pm 0,09$ & $1,88 \pm 0,15^{* *}$ & $2,47 \pm 0,17 * * \#$ & $2,60 \pm 0,16^{* * \#}$ & 0,005 \\
\hline GL, g/l & $5,18 \pm 0,30$ & $7,31 \pm 0,33 * *$ & $8,41 \pm 0,26^{* * \#}$ & $9,54 \pm 0,31^{* *} \#^{\prime \prime}$ & $<0,001$ \\
\hline LDL, unit & $38,5 \pm 2,2$ & $63,6 \pm 5,9 * *$ & $83,6 \pm 7,1 * * \#$ & $96,2 \pm 10,0 * * \#$ & 0,010 \\
\hline HDL, mmol/1 & $1,62 \pm 0,05$ & $1,66 \pm 0,14$ & $1,15 \pm 0,07 * * \#$ & $1,05 \pm 0,08^{* * \#}$ & 0,001 \\
\hline
\end{tabular}

Note: ${ }^{*}-p<0,05 ; * *-p<0,001$ comparing with PHP group; \#-p<0,05 comparing with correspondent group of patients with BMI 25-29,9 kg/m²; " - p<0,05 comparing with correspondent group of patients with BMI 30-34,9 kg/m²; $p_{F}$-level of significances of differences of indices between groups with different BMI in whole by ANOVA univariate disperse analysis

There was a significant increase of fractions of atherogenic LDL that was also fixed in 3 and 2 groups and reached maximal values (96,2 $\pm 10,0$ units and 83,6 $\pm 7,1$ units respectively) with increase in 1,5 and 1,3 times comparing with patients of 1 group and LDL level $-63,6 \pm 5,9$ units ( $p<0,05$ at all comparisons) and with increase in 2,5 and 2,2 times as to PHP ( $p<0,001$ at all comparisons). In patients with NASH, who had BMI $30-34,9 \mathrm{~kg} / \mathrm{m}^{2}$ and $35-39,9 \mathrm{~kg} / \mathrm{m}^{2}$, concentration of antiatherogenic HDL fraction in blood serum was lower $(1,15 \pm 0,07 \mathrm{mmol} / 1$ and 1,05 $\pm 0,08 \mathrm{mmol} / 1$, respectively) comparing with BMI $25-29,9 \mathrm{~kg} / \mathrm{m}^{2}(\mathrm{p}<0,01)$ and PHP $(\mathrm{p}<0,001)$. At that HDL level in patients with BMI $25-29,9 \mathrm{~kg} / \mathrm{m}^{2}$ did not differ from PHP values $(\mathrm{p}>0,05)$.

The direct dependence of indices of lipid profile from BMI is demonstrated by the results of correlative analysis: BMI increase led to the growth of the levels of GCS $\left(r_{s}=0,454, p<0,001\right)$, TG in plasma $\left(r_{s}=0,417, p<0,001\right), \operatorname{LDL}\left(r_{s}=0,393, p<0,001\right), G L\left(r_{s}=0,465, p<0,001\right)$ and reliable lowering of $\operatorname{HDL}\left(\mathrm{r}_{\mathrm{s}}=-0,356, \mathrm{p}<0,001\right)$.

Analysis of distribution of changes of indices of lipid metabolism comparing with norm (Fig. 1) demonstrated that GCS level was increased in $35(94,6 \%)$ patients of 2 group, in all patients of 3 group ( $n=23-100 \%)$ and only in $25(62,5 \%)$ patients from 1 group $(p<0,01)$, that shows an expressed dependence of lipid blood spectrum parameters from BMI indices. Analogous reliable data were observed also relative to the indices of TG, GL and LDL. Maximal number of increased data of TG was in 2 group - $34(91,9 \%)$ and 3 group - $23(100 \%)$ against $24(60 \%)$ cases in 1 group $(\mathrm{p}<0,01)$.

GL increase was typical for 22 (95,7 \%) patients from 3 group, for $25(67,5 \%)$ ones from 2 group that reliably $(\mathrm{p}<0,01)$ increased the correspondent indices in patients of 1 group $(n=13-32,5 \%)$. GL value within physiological norm was observed only in $1(4,3 \%)$ patient from 3 group relative to $12(32,4 \%)$ from 2 group, $\mathrm{p}<0,01$ and $27(67,5 \%)$ from 1 group, $(\mathrm{p}<0,001)$.

LDL level was increased in all patients $23(100 \%)$ from 3 group, in $31(83,8 \%)$ from 2 group comparing with only $15(37,5 \%)$ patients from 1 group $(\mathrm{p}<0,01)$. LDL normal values were observed in 2 group only in $5(13,5 \%)$ against $19(47,5 \%)$ from 1 group $(\mathrm{p}<0,01)$. The opposite tendency of changes was established at analysis of index of HDL content in the blood of patients with NASH in all groups of comparison. Especially, there was registered the reliable decrease of HDL content in most patients from 3 group - in $15(65,2 \%)$ relative to $16(43,2 \%)$ patients from 2 group and only to $5(12,5 \%)$ from 1 group $(\mathrm{p}<0,01)$. 


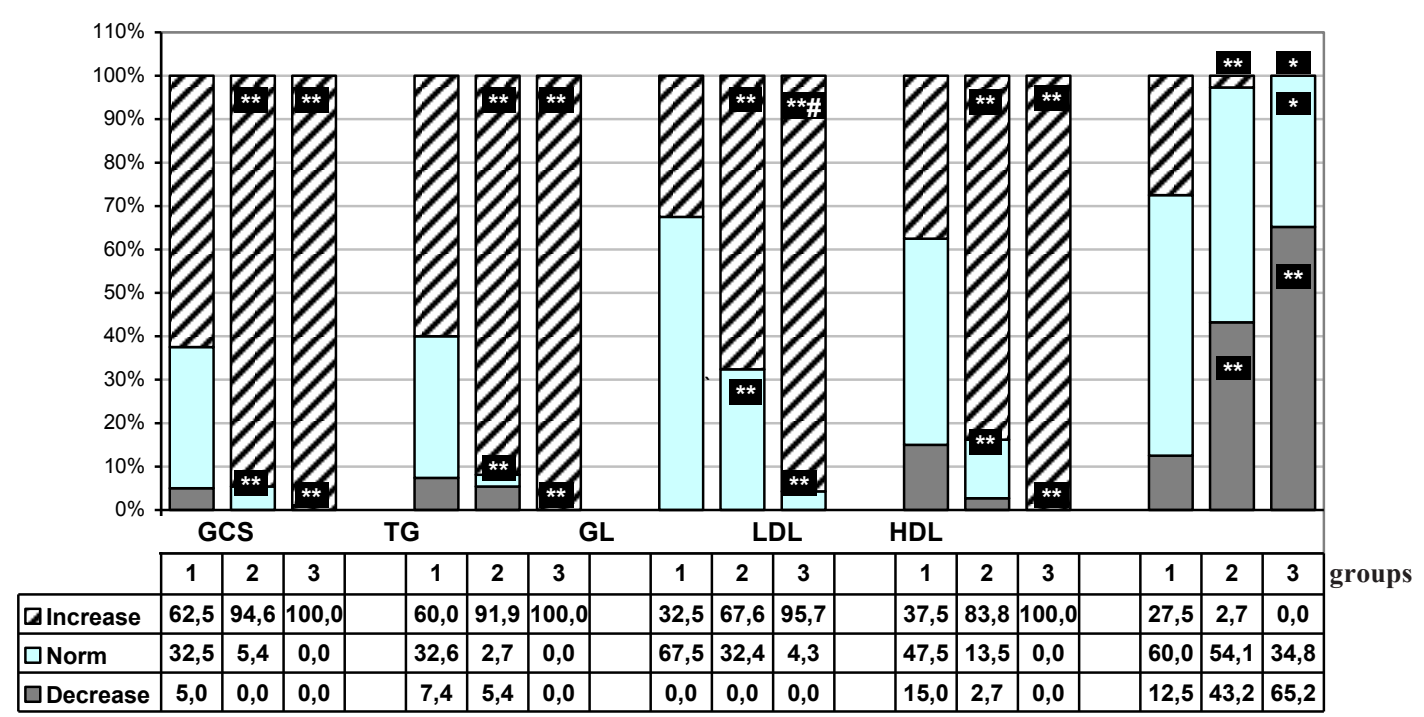

Fig. 1. Distribution of changes of the lipid metabolism parameters comparing with norm in patients with non-alcoholic steatohepatitis in combination with obesity depending of BMI:

* $-\mathrm{p}<0,05 ;{ }^{* *}-\mathrm{p}<0,01$ comparing with correspondent group of patients with BMI $25-29,9 \mathrm{~kg} / \mathrm{m}^{2}$; $\#-\mathrm{p}<0,05$ comparing with correspondent group of patients with BMI $30-34,9 \mathrm{~kg} / \mathrm{m}^{2}$

At detail consideration of lipid profile indices in patients with NASH in combination with obesity depending on concomitant BT pathology (Table 2) analogical tendencies were revealed and they were expressed by all indices of lipid spectrum. Thus, GCS content $-7,18 \pm 0,38 \mathrm{mmol} / 1$ exceeded PHP value by $37,3 \%$ in patients with concomitant CNC, by $49,7 \%$ in patients with CCC and by $40,7 \%$ at PCES ( $<<0,001$ at all comparisons). TG indices also were almost equally increased in all groups of observation with maximal increase in 2,1 times as to PHP in patients with PCES, and in 1,9 times in patients with concomitant $\mathrm{CNC}$ and $\mathrm{CCC}(\mathrm{p}<0,001$ at all comparisons).

Table 2

Comparative characteristic of indices of lipid metabolism in patients with non-alcoholic steatohepatitis in combination with obesity depending on concomitant pathology of biliary tract $(\mathrm{M} \pm \mathrm{m})$

\begin{tabular}{cccccc}
\hline \multirow{2}{*}{ Index } & PHP, $\mathbf{n = 2 0}$ & With $\mathbf{C N C}, \mathbf{n}=\mathbf{3 5}$ & With $\mathbf{C C C}, \mathbf{n}=\mathbf{3 0}$ & With PCES, $\mathbf{n = 3 5}$ & $\mathbf{p}_{\mathbf{F}}$ between groups \\
\hline GCS, mmol/1 & $5,23 \pm 0,17$ & $7,18 \pm 0,38^{*}$ & $7,83 \pm 0,55^{*}$ & $7,36 \pm 0,54^{*}$ & 0,639 \\
TG, mmol/1 & $1,14 \pm 0,09$ & $2,15 \pm 0,19^{*}$ & $2,21 \pm 0,16^{*}$ & $2,42 \pm 0,16^{*}$ & 0,486 \\
GL, g/l & $5,18 \pm 0,30$ & $8,09 \pm 0,29^{*}$ & $8,04 \pm 0,43^{*}$ & $8,53 \pm 0,32^{*}$ & 0,540 \\
LDL, unit & $38,5 \pm 2,2$ & $80,2 \pm 7,3^{*}$ & $83,6 \pm 9,6^{*}$ & $72,4 \pm 6,0^{*}$ & 0,565 \\
HDL, mmol/1 & $1,62 \pm 0,05$ & $1,16 \pm 0,08^{*}$ & $1,19 \pm 0,10^{*}$ & $1,63 \pm 0,16$ & 0,009
\end{tabular}

Note * $-p<0,001$ comparing with PHP group; \# $-p<0,05$ comparing with correspondent group of patients with PCES; $p_{F}$-level of significance of difference of indices between groups with NASH in whole by ANOVA univariate disperse analysis

GL content was also increased in all patients with NASH, in 1,6 times at CNC and CCC and in 1,7 times in patients with concomitant PCES ( $p<0,001$ at all comparisons). LDL values were increased in 1,9 times at concomitant PCES and in 2,1 and 2,2 times respectively in patients with concomitant $\mathrm{CNC}$ and $\mathrm{CCC}$ ( $\mathrm{p}<0,001$ at all comparisons). HDL was equally decreased in groups with concomitant $\mathrm{CNC}$ and $\mathrm{CCC}$ in 1,4 times $(\mathrm{p}<0,001)$ and did not differ from PHP indices in patients with concomitant PECS that indicates to the less inflammatory activity in BT after LCE. This point of view is proved also by the less intergroup increase of LDL in patients with concomitant PCES. 
Revelation of deviations of the lipid metabolism indices depending on the limit of physiological norm in patients with NASH in combination with obesity taking into account BT pathology is presented on the Fig. 2. According to the data of Fig. 2, the raise of FCS, TG, GL and LDL was observed with equal frequency in all groups of observation. The reliably significant changes of HDL level at the expense of their decrease were typical for patients with concomitant PCES and were observed in $8(22,9 \%)$ against $17(48,6 \%)$ in patients with concomitant $\mathrm{CNC}(\mathrm{p}<0,05)$.

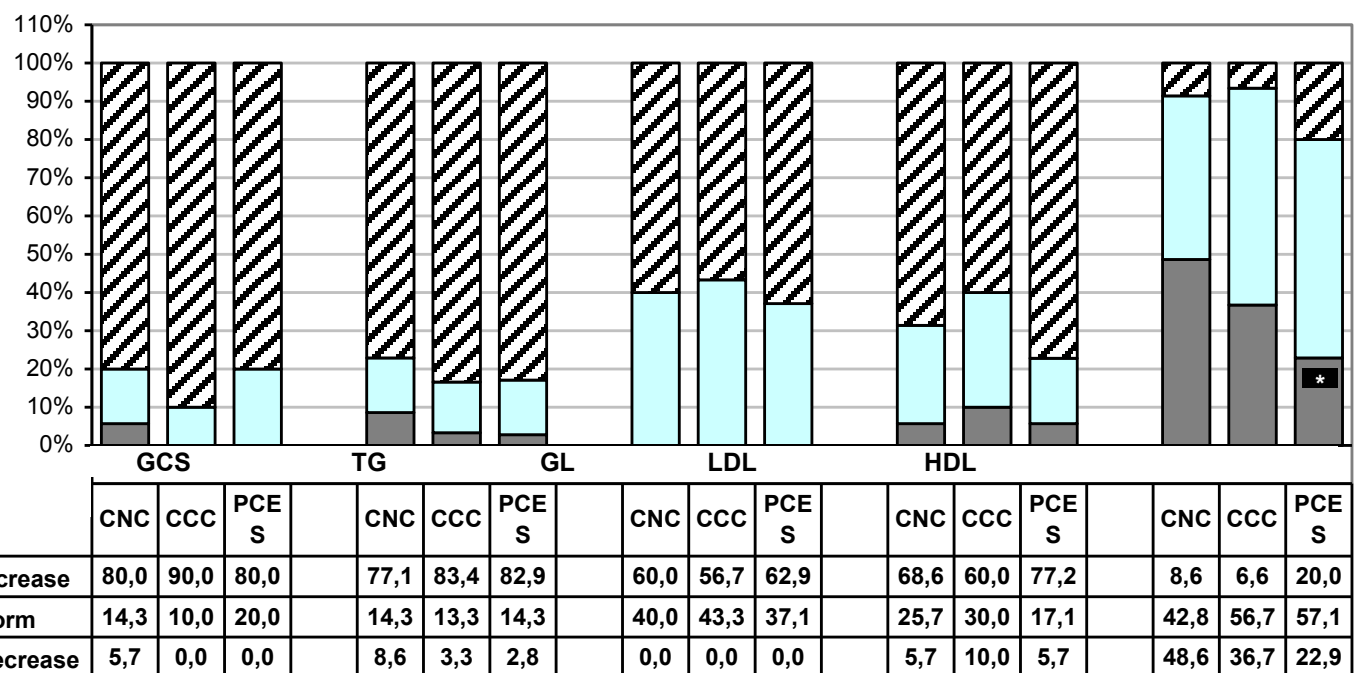

Fig. 2. Distribution of changes of lipid metabolism indices comparing with norm in patients with non-alcoholic steatohepatitis in combination with obesity depending on concomitant pathology of

biliary tract: $*_{-} \mathrm{p}<0,05$ comparing with correspondent group of patients with CNC

Analysis of frequency of revelation of the main dyslipidemia phenotypes among patients with NASH in combination with obesity and BT pathology depending on BMI is presented in the Table 3 and Fig. 3.

Table 3

Frequency of revelation of the main dyslipidemia phenotypes among patients with non-alcoholic steatohepatitis in combination with obesity and pathology of biliary tract depending on BMI

\begin{tabular}{|c|c|c|c|c|}
\hline & \multirow{3}{*}{ Groups } & \multicolumn{3}{|c|}{ DL presence/absence } \\
\hline & & \multirow{2}{*}{ Absence of DL } & \multicolumn{2}{|c|}{ DL phenotype } \\
\hline & & & IIa & IIb \\
\hline \multirow{4}{*}{$\mathrm{NASH}, \mathrm{n}=100$} & $\begin{array}{c}\text { BMI-1.2 } \\
\left(25-29,9 \mathrm{~kg} / \mathrm{m}^{2}\right), \mathrm{n}=40\end{array}$ & $25(62,5 \%)$ & $4(10,0 \%)$ & $11(27,5 \%)$ \\
\hline & $\begin{array}{c}\text { BMI- } 2.2 \\
\left(30-34,9 \mathrm{~kg} / \mathrm{m}^{2}\right), \mathrm{n}=37\end{array}$ & $6(16,2 \%)^{*}$ & $5(13,5 \%)$ & $26(70,3 \%)^{*}$ \\
\hline & $\begin{array}{c}\text { BMI-3.2 } \\
\left(35-39,9 \mathrm{~kg} / \mathrm{m}^{2}\right), \mathrm{n}=23\end{array}$ & $-*$ & $5(21,7 \%)$ & $18(78,3 \%)^{*}$ \\
\hline & $\mathrm{p}_{x^{2}}$ between groups & $<0,001$ & 0,431 & $<0,001$ \\
\hline
\end{tabular}

Note: * ${ }^{*}<0,001$ comparing with correspondent group of patients with BMI 25-29,9 kg/ $\mathrm{m}^{2} ; p_{x^{2}}$ - level of significance of differences between groups with different BMI in whole by $\chi^{2}$ criterion

According to the table data, at NASH reliable changes were detected relative to the patients with BMI 25-29,9 kg/m² ( $<<0,001)$. Thus, IIb phenotype was observed in $26(70,3 \%)$ patients with BMI $30-34,9 \mathrm{~kg} / \mathrm{m}^{2}$ and in $18(78,3 \%)$ with BMI 35-39,9 kg/m² against $11(27,5 \%)$ at BMI $25-29,9 \mathrm{~kg} / \mathrm{m}^{2}$ $(\mathrm{p}<0,001)$. The absence of DL was fixed only in $6(16,2 \%)$ patients with BMI $30-34,9 \mathrm{~kg} / \mathrm{m}^{2}$ and was not observed at all at BMI 35-39,9 $\mathrm{kg} / \mathrm{m}^{2}$ relative to patients with BMI $25-29,9 \mathrm{~kg} / \mathrm{m}^{2}-$ in 25 $(62,5 \%)(\mathrm{p}<0,001)$. 


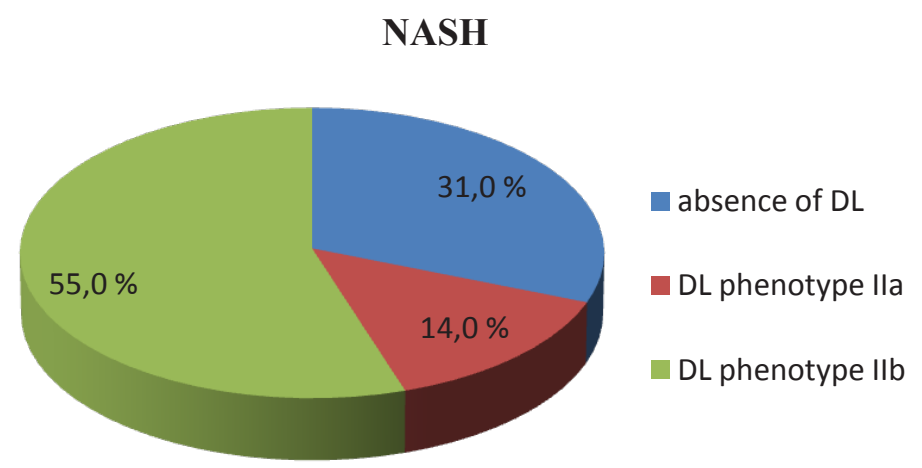

Fig. 3. Distribution of dyslipidemia types in patients with NASH in combination with obesity and BT pathology

\section{Discussion}

In patients with NASH in combination with obesity and BT pathology the disorders of lipid metabolism play one of the main roles in the formation of metabolic status. There were established the features of changes of the lipid blood spectrum in this category of patients that are manifested in variability of disorders from hypercholesterolemia to hypertriglyceridemia on the background of increase of LDL and decrease of HDL. The received data of comparative evaluation of lipid parameters of patients with NASH in combination with obesity and BT pathology testify that TG play an important role in formation of lipoproteins, increase of GL and LDL content in patients with NASH.

The decrease of intensity of reverse transport of GCS from tissues to liver as a result of insufficient synthesis of HDL and essential level of hypercholesterolemia in LDL content and hypertriglyceridemia create conditions not only for development and progression of NAFDL but also for the vascular disorders, especially, atherosclerotic injury of the main vessels and the ones of liver and create conditions for formation of the vascular component of NAFDL pathogenesis and its progression.

The received data testify that progression of intensity of lipid disorders in patients with NASH in combination with obesity and BT pathology depend on increase of BMI parameters. Analysis of lipid changes in patients with NASH demonstrated the reliable increase of GCS with parallel raise of TG, GL, LDL, fixed at growth of BMI relative to PHP (from $\mathrm{p}<0,05$ to $\mathrm{p}<0,001$ ) and patients of the other groups $(\mathrm{p}<0,05)$. The highest indices of GCS, TG, GL, LDL were in patients with NASH and obesity of I degree $(p<0,001)$ and obesity of II degree $(p<0,001)$, and the least heightened parameters of the lipid blood spectrum, relative to PHP, at BMI $(p<0,05 ; p<0,001)$. Analogous changes but with decrease of HDL were also fixed at obesity of I and II degree in patients of groups of NASH relative to PHP $(p<0,05)$. Along with it, at EBM HDL content did not differ from PHP values ( $p>0,05$ ). It is possible to assume that BMI changes on the background of its growth act as independent additional factor of progression of NASH in combination with obesity and also favor the development and progression of other metabolic disorders.

On the background of received data, depending on concomitant BT pathology is obvious that the lipid metabolism disorders were observed in all groups of patients with NASH in combination with obesity, were characterized with hypertriglyceridemia and disorder of metabolism and transport of CS, that were more intense in patients with NASH and concomitant CNC and CCC at the lowered HDL level. The presence of concomitant pathology from BT side partially influenced the indices of lipid blood spectrum. Thus, the low level of HDL in patients with NASH in combination with obesity and concomitant $\mathrm{CNC}$ anc $\mathrm{CCC}$ can be explained by the intense inflammatory processes in biliary tract, attended with disorders of indices of lipid profile with decrease of HDL and increase of CS, TG, GL and LDL.

The received data testify that at NASH with increased BMI the DL phenomena take place more and more often. There were separated two main phenotypes of dyslipidemia: IIa Ta IIb, which 
comparing with nosology and stage of disease of patients allowed consider them as the main phenotypes, typical for the studied comorbid pathology. The second phenotype of dyslipidemia is manifested as a complex of biological defects of lipid metabolism as moderate hyperlipidemia and is typical for patients with NASH.

In further works it is planned to study the lipid-phospholipid disorders, depending on BMI and reveal their influence on progression of the main disease in patients with comorbid course of non-alcoholic fatty disease of liver and obesity on the background of concomitant pathology of biliary tract.

\section{Conclusions}

1. Progression of intensity of lipid disorders in patients with NASH in combination with obesity and BT pathology depends on increase of BMI parameters. The most indices of GCS, TG, GL, LDL were in patients with NASH at obesity of I degree $(p<0,001)$ and obesity of II degree $(p<0,001)$, and the lowest parameters of lipid blood spectrum, relative to PHP, at $\operatorname{EBM}(\mathrm{p}<0,05, \mathrm{p}<0,001)$.

2. Concomitant pathology from BT side partially influenced the indices of lipid blood spectrum. Thus, HDL were equally decreased in patients with NASH at concomitant CNC and CCC relative to the norm $(\mathrm{p}<0,001)$ and HDL level within physiological norm in patients with concomitant PCES $(\mathrm{p}>0,05)$.

3. At NASH in combination with obesity with BMI increase the DL phenomena appear in patients more often. There were two main phenotypes of dyslipidemia: IIа та IIb. Is was established, that IIb phenotype of dyslipidemia was most often observed in patients with BMI $30-34,9 \mathrm{~kg} / \mathrm{m}^{2}$ and $35-39,9 \mathrm{~kg} / \mathrm{m}^{2}$ as to patients with BMI $25-29,9 \mathrm{~kg} / \mathrm{m}^{2}(\mathrm{p}<0,001)$.

\section{References}

[1] Bobronnikova, L. R., Zhuravlyova, A. K. (2013). The mechanisms of progression of dyslipidemia in patients with nonalcoholic fatty liver disease. Ukrainian therapeutical journal, 2 (37), 83-88.

[2] Stepanov, Yu. M., Filippova, A. Yu. (2013). Nonalcoholic fatty liver disease and atherogenic dyslipidemia (modern approaches to diagnosis and treatment). Kyiv: Metodicheskie rekomendatsii, 36.

[3] Stepanov, Yu. M., Filippova, A. Yu. (2010). Dyslipidaemia pattern in patients with non-alcoholic steatosis and biliary pathology and methods of its correction. Contemporary gastroenterology, 4 (54), 46-51.

[4] Kuryata, O. V., Grechanyk, M. M. (2014). Interrelation of blood lipid spectrum, level of systemic inflammation and mass of body in patients with coronary heart disease in combination with nonalcoholic fatty liver disease and their dynamics in terms of secondary prevention. Medical perspectives, 4 (XIX), 103-111.

[5] Drapkina, O. M., Popova, I. R. (2012). The role of obesity in the development of hypertension and non-alcoholic fatty liver disease. Consilium Medicum, 12, 72-76.

[6] Chatrath, H., Vuppalanchi, R., Chalasani, N. (2012). Dyslipidemia in Patients with Nonalcoholic Fatty Liver Disease. Seminars in Liver Disease, 32 (01), 022-029. doi: 10.1055/s-0032-1306423

[7] Hurjui, D. M., Nita, O., Graur, L. I., Micalache, L. (2012). Non-alcoholic fatty liver disease is associated with cardiovascular risk factors of metabolic syndrome. Rev. Med. Chir. Soc. Med. Nat. Lasi., 116 (3), 692-699.

[8] El-Kader, S. M. A. (2015). Non-alcoholic fatty liver disease: The diagnosis and management. World Journal of Hepatology, 7 (6), 846-858. doi: 10.4254/wjh.v7.i6.846

[9] Novakovic, T., Mekic, M., Smilic, L., Smilic, T., Ini, B., Kostic, K. et. al. (2014). Anthropometric and Biochemical Characteristics of Patients with Nonalcoholic Fatty Liver Diagnosed by Non-Invasive Diagnostic Methods. Medical Archives, 68 (1), 22-26. doi: 10.5455/medarh.2014.68.22-26

[10] Dietrich, P., Hellerbrand, C. (2014). Non-alcoholic fatty liver disease, obesity and the metabolic syndrome. Best Practice \& Research Clinical Gastroenterology, 28 (4), 637-653. doi: 10.1016/ j.bpg.2014.07.008

[11] Kalsch, J., Bechmann, L. P., Kalsch, H., Schlattjan, M., Erhard, J., Gerken, G., Canbay, A. (2011). Evaluation of Biomarkers of NAFLD in a Cohort of Morbidly Obese Patients. Journal of Nutrition and Metabolism, 2011, 1-7. doi: 10.1155/2011/369168

[12] Ilchenko, A. A. (2011). Postcholecystectomy syndrome: a look at the problem from the perspective of the therapist. Annals of surgical Hepatology, 2 (16), 37-44. 
[13] Order of HM from Ukraine 13.06.2005 N 271. On approval of the protocols of medical care to patients with a degree in Gastroenterology.

[14] Order of HM from Ukraine 06.08.2014 N 826. Unified clinical protocols of primary, secondary (specialized) medical care "Nonalcoholic steatohepatitis".

[15] Toouli, J., Fried, M., Ghafoor, A. (2009). Obesity World Gastroenterology Organisation Global Guideline, 30 .

[16] Hashizume, H., Sato, K., Yamazaki, Y. (2013). A prospective study of long-term outcomes in female patients with non-alcoholic steatohepatitis using age- and body mass index-matched cohorts. Acta Med Okayama, 67, 45-53.

[17] Muller, M. J., Lagerpusch, M., Enderle, J., Schautz, B., Heller, M., Bosy-Westphal, A. (2012). Beyond the body mass index: tracking body composition in the pathogenesis of obesity and the metabolic syndrome. Obesity Reviews, 13, 6-13. doi: 10.1111/j.1467-789x.2012.01033.x

[18] Kamyishnikov, V. S. (2014). Clinical and laboratory diagnosis of liver diseases. $2^{\text {nd }}$ edition. Moscow: MEDpress-inform.

[19] ESC/EAS Guidelines for the management of dyslipidaemias. The Task Force for the management of dyslipidaemias of the European Society of Cardiology (ESC) and the European Atherocklerosis Society (EAS) (2011). Eur. Heart J., 32, 1769-1818.

[20] Gromov, A. I., Rybczynski, S. S. (2009). The Value of ultrasound in the diagnosis of fatty hepatosis. Clinical medicine, 8, 64-66.

[21] Hernaez, R., Lazo, M., Bonekamp, S., Kamel, I., Brancati, F. L., Guallar, E., Clark, J. M. (2011). Diagnostic accuracy and reliability of ultrasonography for the detection of fatty liver: A meta-analysis. Hepatology, 54 (3), 1082-1090. doi: 10.1002/hep.24452 